\title{
Investigation of Defect Nucleation in Titanium under Mechanical Loading
}

\author{
Konstantin P. Zolnikov, 2, a), Aleksandr V. Korchuganov', \\ Dmitrij S. Kryzhevich ${ }^{1,2}$, and Sergey G. Psakhie ${ }^{1,3,4}$ \\ ${ }^{1}$ Institute of Strength Physics and Materials Science SB RAS, Tomsk, 634055, Russia \\ ${ }^{2}$ National Research Tomsk State University, Tomsk, 634050, Russia \\ ${ }^{3}$ National Research Tomsk Polytechnic University, Tomsk, 634050, Russia \\ ${ }^{4}$ Skolkovo Institute of Science and Technology, Skolkovo, 143025, Russia \\ a) Corresponding author: kost@ispms.ru
}

\begin{abstract}
The paper undertakes a study of plastic deformation in a titanium crystallite under mechanical loading (uniaxial tension and indentation) in terms of atomic mechanisms of its generation and development. The molecular dynamics method with many-body interatomic potentials is employed. It is shown that there is a threshold strain, at which a crystal reveals the generation of local structural transformations associated with changes in atomic configurations of the first and second coordination spheres. The onset of plastic deformation in a crystallite is accompanied by a stepwise decrease in potential energy. The effect of free surfaces and grain boundaries on the generation of local structural transformations in a titanium crystallite is investigated.
\end{abstract}

Keywords: nanoindentation, molecular dynamics, structural defects

\section{INTRODUCTION}

Structural transformations in materials under the external actions present a complex process associated with the nucleation and evolution of structural defects at different scales [1]. The study of atomic mechanisms responsible for the generation of plastic deformation is an important fundamental problem of the solid state physics and contemporary materials science. This is explained by the fact that plasticity originate at the micro (atomic) scale and govern the development of a defect structure at higher scales. Formation of structural defects allows a partial compensation of elastic fields induced by external actions in the material and thereby a reduction of elastic energy of the material. Despite significant advances in the study of structural response of various materials under mechanical action, the problem of generation of plastic deformation at the atomic scale has not been adequately explored and is studied further in many theoretical and experimental works [2-6]. Characteristic times and scales used to describe local structural transformations are extremely small (picoseconds and angstroms, respectively). This makes the experimental study of the generation and development of plastic deformation at the atomic scale difficult. An efficient approach to solving this problem is computer simulation, particularly the molecular dynamics method. In this connection, the present paper aims at the molecular dynamics investigation of atomic mechanisms of the generation and development of structural transformations in a titanium crystallite with consideration for free surfaces and grain boundaries at different types of mechanical loading (uniaxial tension and nanoindentation).

\section{MATERIALS AND METHODS}

The set problem has been solved by the molecular dynamics method using many-body interatomic potentials obtained in the framework of the embedded atom method [7]. Used potentials allow describing elastic energetic and structural properties of the simulated crystallite to a high degree of accuracy with the proper account of their

International Conference on Physical Mesomechanics of Multilevel Systems 2014

AIP Conf. Proc. 1623, 675-678 (2014); doi: 10.1063/1.4899035

(C) 2014 AIP Publishing LLC 978-0-7354-1260-6/ $\$ 30.00$ 
properties near interfaces. This approach provides an explicit consideration of the discrete structure of the simulated material and adequate description of the behavior of its atomic system.

Simulated titanium crystallites are rectangular parallelepipeds. Their equilibrium lattice parameters are $a=2.97$ and $c=4.68 \AA$. Rigid boundary conditions are assigned at the two opposite faces of the simulated crystallite. The other two faces of the specimen are free surfaces while the third direction is imposed with periodic boundary conditions. Rigid boundary conditions are used to simulate uniaxial tension of the crystallite with constant velocity (Fig. 1). The tensile velocity ranges from 5 to $45 \mathrm{~m} / \mathrm{s}$. The kinetic temperature of the studied specimen before loading is $T=100 \mathrm{~K}$.

\section{UNIAXIAL TENSION}

The computer experiments show that in tension the potential energy is monotonically accumulated in a crystallite. As this takes place, at a certain threshold strain the specimen reveals the generation of local structural changes, which causes potential energy to grow slower. Note that local structural changes appear in an avalanche manner in the simulated crystallite. Such behavior of the material results in a rather rapid decrease of the potential energy of the specimen (Fig. 1). Similarly to other materials $[8,9]$, threshold strain depends on loading rate, temperature of the simulated specimen, presence of extended interfaces in it, etc.

To analyze the generation and development of plastic deformation in simulated titanium crystallites, calculation of the reduced slip vector has been made:

$$
P_{i}=\frac{1}{N_{s}} \sum_{i \neq j}^{N_{s}} \frac{r^{i j}-r_{0}^{i j}}{\left|r_{0}^{i j}\right|},
$$

where $j$ is the nearest neighbor of atom $i, N_{s}$ is the number of nearest neighbors, $r^{i j}$ and $r_{0}^{i j}$ are the radius vectors of the $j$-th atom with regard to the $i$-th one in the current and initial positions, respectively.

To study behavioral peculiarities of the atomic structure during crystallite deformation the analysis of atomic displacements at different reduced slip vectors is performed. Note that the identification of local structural changes through the slip vector requires respective thresholds to be found. Thus, the distance to nearest neighbors is set as average radii of the first and second coordination spheres in the ideal lattice of titanium. According to the analysis of the simulated results, the threshold value of $P_{i}$ separating elastic displacements of atoms from lattice distortions induced by plastic deformation is assumed to equal 0.15 .

The strain dependence of the potential energy of the crystallite and number of atoms with different reduced slip vectors $P_{i}$ is shown in Fig. 1 .

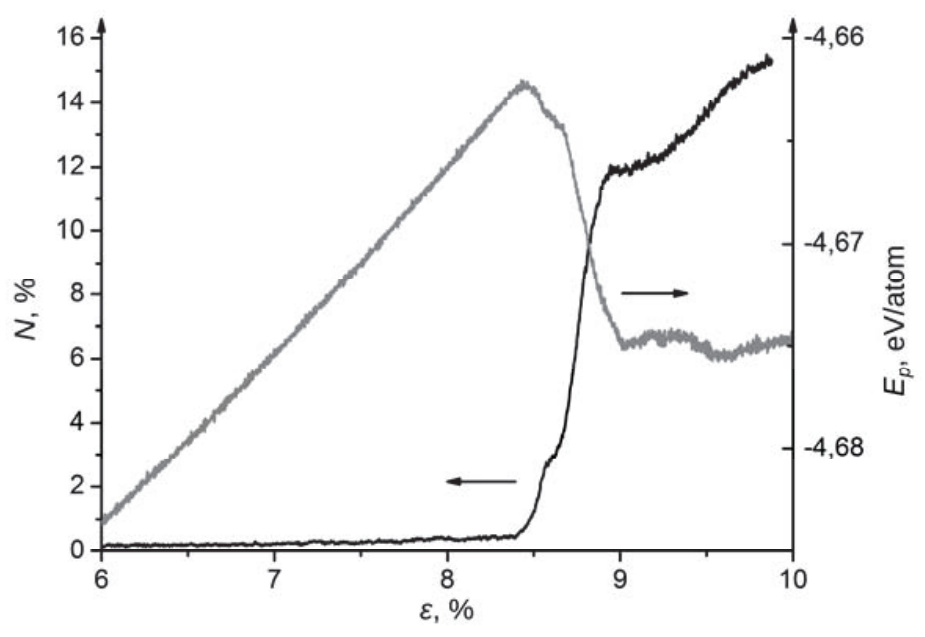

FIGURE 1. Strain $\varepsilon$ versus the number of atoms $(N)$ with $P_{i}$ more than 0.15 (black curve) and versus potential energy $\left(E_{\mathrm{p}}\right.$, gray curve) 


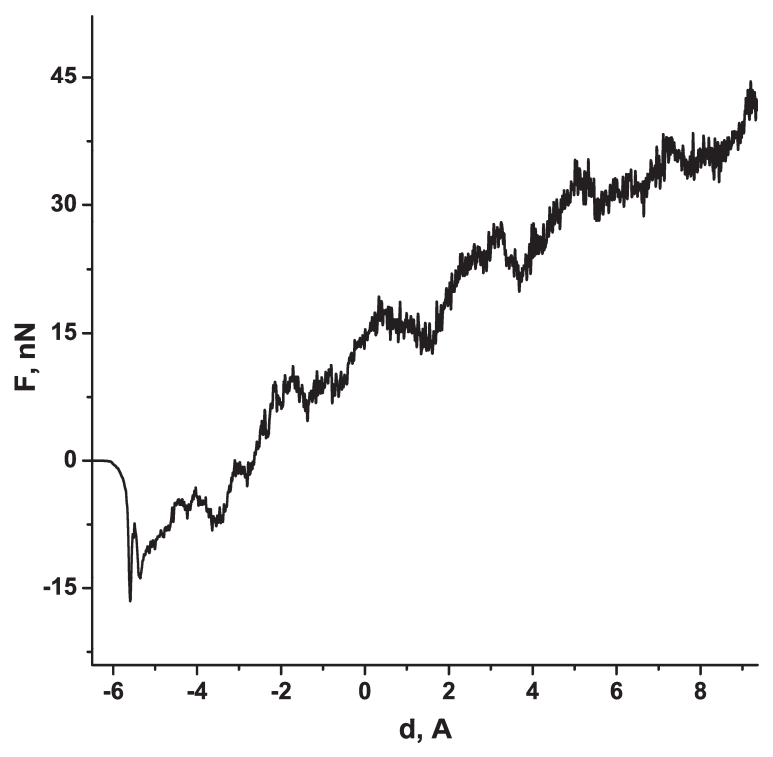

FIGURE 2. Reaction force of the crystallite versus indentation depth

At the strain $\sim 8.4 \%$ the curve for potential energy per atom (gray curve) is seen to vary considerably in shape, namely, its slope angle decreases to a negative value. In this case the number of atoms with an elevated slip vector begins to grow (black curve). The figure shows that the slope angle variation for the potential energy curve correlates with that of the curve for the number of atoms with an elevated slip vector. This is explained by the fact that the formation of local structural changes in the titanium crystallite is a way of relaxation to mechanical loading.

\section{NANOINDENTATION}

The study of the material behavior at indentation makes is possible to control load localization. Of great significance is the selection of configuration of the simulated system and loading conditions. To simplify the analysis of the simulation results loading is performed by an elongated semicylindrical indenter. With such an indenter the loading area is extended from one to another face of the simulated specimen. The indenter was a perfectly rigid body made of titanium atoms with fixed positions relative to each other.

The simulated specimen has a parallelepiped shape. Periodic boundary conditions are set along the indenter axis while free boundary conditions are simulated at the side faces. The loading surface of the crystallite is free while the opposite one is imposed with rigid boundary conditions. The indenter moves with a constant velocity of $25 \mathrm{~m} / \mathrm{s}$. The kinetic temperature of the specimen is $300 \mathrm{~K}$.

The calculation results for the reaction force of the specimen as a function of indentation depth are presented in Fig. 2. As consisted of titanium atoms, the indenter approaches the crystallite surface with arising force of mutual attraction. This corresponds to negative values of the force in Fig. 2. Note that the curve in this figure is characterized by numerous "small" oscillations and large enough kinks. They result from thermal fluctuations of the atomic system and discrete nature of the simulated structure. In particular, the discrete structure of the indenter and crystallite has a significant influence on the reaction force especially at the initial moment (area of mutual attraction). This is due to the fact that the atomic layer of the indenter tip is the first to experience attraction, which is followed by its subsequent layers. As atoms of the indenter tip are already under the action of repulsive force from the crystallite, its sides are just coming into attraction to the crystallite surface, which undoubtedly affects the curve behavior. Kinks of the curve in Fig. 2 demonstrate a pronounced periodicity, which correlates well with the interplanar spacing in the crystal in the direction of indentation. The analysis of the calculation results suggests that at the indentation depth $\sim 4.5 \AA$ stacking faults are beginning to nucleate in the loaded crystallite. They appear initially under the indenter and then propagate in the glide plane (0001) in the direction of the side face. Their occurrence at the side face causes a step to form at the free surface. 


\section{CONCLUSIONS}

The calculations show that at a certain threshold strain the titanium crystallite reveals redistribution of elastic energy. This process is related to the generation and avalanche-like growth of local structural changes in the material. Nucleation of defects leads to a much slower increase in the potential energy and its subsequent stepwise decrease to partly compensate elastic stresses induced by crystal deformation. Further loading of the simulated crystallite results in the generation of usual structural defects, such as stacking faults based on local structural changes.

The discrete structure of the simulated system is proved to significantly affect the behavior of the titanium crystallite at nanoindentation. This is reflected in periodic kinks in the curve for the crystallite reaction force versus indentation depth. The nucleation and propagation of defects in the crystal is found to decrease the slope of the above dependence. Such behavior is explained by the fact that the generation of stacking faults is a way of relaxation of elastic stresses. The occurrence of stacking faults at the free surface causes its shape-changing.

Section 2 is partially supported by the TSU and TPU competitiveness enhancement programs. Section 3 is

performed in the framework of the Program of Basic scientific research of the State academies of sciences for 20132020.

\section{REFERENCES}

1. V. E. Panin and V. E. Egorushkin, Phys. Mesomech. 14(5-6), 207 (2011).

2. F. Sansoz and V. Dupont, Mat. Sci. Eng. C 27, 1509 (2007).

3. R. Gröger and V. Vitek, Acta Mater. 56, 5426 (2008).

4. P. Liu, S. C. Mao, L. H. Wang, X. D. Han, and Z. Zhang, Scripta Mater. 64, 343 (2011).

5. K. P. Zol'nikov, T. Yu. Uvarov, and S. G. Psakh'e, Tech. Phys. Lett. 27, 263 (2001).

6. S. G. Psakhie, S. Y. Korostelev, S. I. Negreskul, K. P. Zolnikov, Zhongguang Wang, and Shouxin Li, Phys. Status Solidi B 176, 41 (1993).

7. R. G. Hennig, T. J. Lenosky, D. R. Trinkle, S. P. Rudin, and J. W. Wilkins, Phys. Rev. B 78, 054121 (2008).

8. K. P. Zol'nikov, T. Yu. Uvarov, and S. G. Psakh'e, Tech. Phys, Lett. 27, 263 (2001).

9. S. G. Psakhie, K. P. Zolnikov, D. S. Kryzhevich, and A. G. Lipnitskii, Phys. Lett. A 349, 509 (2006). 